\title{
A GNSS Interference Detection Method Based on Multiple Ground Stations
}

\author{
Sun Young Kim¹, Chang Ho Kang', Jeong Hwan Yang', Chan Gook Park ${ }^{1,2 \dagger}$, Jung Min Joo ${ }^{3}$, Moon Beom $\mathrm{HeO}^{3}$ \\ ${ }^{1}$ School of Mechanical \& Aerospace Engineering, Seoul National University, Seoul 151-742, Korea \\ ${ }^{2}$ Automation and Systems Research Institute, Seoul National University, Seoul 151-742, Korea \\ ${ }^{3}$ Korea Aerospace Research Institute, Daejeon 305-806, Korea
}

\begin{abstract}
For a GNSS receiver's robustness against RFI and the high accuracy of navigation solution in GNSS, interference source detection and mitigation are needed. In this paper, an adaptive lattice IIR notch filter is employed to track single-tone continuous wave and swept continuous wave interference signals, and an interference detection method is proposed. Furthermore, this paper presents interference source characterization algorithm using multiple ground stations' interference detection results. The measurement of the signal powers from each ground station is used to build weighting factors to estimate the type of the interference. The performance of interference detection algorithm is simulated for scenarios of GPS signal in the presence of single-tone continuous wave interference and swept continuous wave interference.
\end{abstract}

Keywords: GNSS, interference detection, adaptive notch filter

\section{INTRODUCTION}

As the use of GNSS increases, integrity monitoring methods have been studied to provide high precision of GNSS positioning solutions. There are many factors that influence the accuracy of GNSS positioning solutions, but the main source of them is intentional interference which intentionally damages the operation of the GNSS system. For example, the performance of a GNSS receiver will be degraded as the power level of the interference signal becomes higher than the receiver's anti-jamming ability. The interference can easily overwhelm a GNSS receiver's analog-to-digital converter at the analog front-end module and paralyze the GNSS receiver. The previous work (Betz 2000) presented that one of the most effective interference sources is the continuous wave interference (CWI). For this reason, CWI detection and mitigation method by adaptive filtering technique have attracted great attention.

Detection and mitigation methods of the CWI by using

Received Aug 30, 2012 Revised Oct 01, 2012 Accepted Oct 02, 2012

†Corresponding Author

E-mail: chanpark@snu.ac.kr

Tel: +82-2-880-1732 Fax: +82-2-873-1732 the adaptive filter have been studied recently and can be classified into two approaches: time-domain (Cho \& Lee 1993, Choi \& Cho 2002, Borio et al. 2008, Chien et al. 2010) and frequency-domain approaches (Capozza et al. 2000, Balaei \& Dempster 2009). These approaches have a limitation on detection and mitigation of swept continuous wave interference, because its sweep rate degrades the signal tracking performance of the adaptive notch filter.

In this paper, an adaptive lattice IIR notch filter (Cho \& Lee 1993, Choi \& Cho 2002, Kang et al. 2012) is implemented to identify the types of interference such as single-tone continuous wave and swept continuous wave signals. The performance of the interference detection and identification method is analyzed using software GPS receiver and interference simulator data. The proposed characterization algorithm is also simulated with a set of three ground stations. Through theoretical analysis and simulation results, performance of the proposed method is shown in this paper.

The rest of this paper is organized as follows: Section 2 refers to the intermediate frequency model of GPS and interference. In Section 3, the adaptive lattice IIR notch filter is briefly reviewed. In addition, the detection method is proposed and is applied in multiple ground stations. 
In Section 4, the performance of the proposed method is applied to the single ground station and multiple ground stations system for evaluating its performance. Finally, conclusions are given in Section 5.

\section{TYPES OF GNSS CONTINUOUS WAVE INTERFERENCE}

The interference is classified into narrowband interference and wideband interference according to the frequency band of the interference. The criterion of narrowband and wideband is determined by the bandwidth of GNSS signal. In this paper, the basis of the bandwidth is selected by 2.046 MHz which is the bandwidth of GPS L1 C/ A code signal. The narrowband interference is defined as its bandwidth is lower than $2.046 \mathrm{MHz}$, and otherwise the interference is defined as wideband interference. This paper is focused on the narrowband interference which affect GPS L1 C/A code signal. In general, the narrowband CWI can be classified into single-tone CWI and swept CWI according to the interference scenario.

The single-tone CWI which has a fixed carrier frequency is modeled as

$$
i_{c w i}(n)=\sqrt{2 P_{i}} \cos \left(2 \pi f_{i} n+\phi_{i}\right)
$$

where $P_{i}$ is the interference signal power, $\phi_{i}$ is the phase, $f_{i}$ is the interference center frequency of $9.548 \mathrm{MHz}$, and $n=t / f_{s}$. In addition, $t$ indicates time, and $f_{s}$ is the sampling frequency of $38.192 \mathrm{MHz}$.

The swept CWI is the interference that changes its center frequency and can be modeled as

$$
i_{\text {swept cwi }}(n)=\sqrt{2 P_{i}} \cos \left(2 \pi f_{i}(n) n+\phi_{i}\right)
$$

where $f_{i}(n)$ adjusts sweep rate and bandwidth, and is modeled as, $f_{i}(n)=\left(f_{I F}-\right.$ bandwidth $\left./ 2\right)+n \times$ sweep rate. In this paper, the bandwidth is set by $4 \mathrm{MHz}$, and the change of sweep rate is set to $100 \sim 500 \mathrm{MHz} / \mathrm{sec}$.

The frequency domain response of the single-tone CWI and swept CWI are shown in Fig. 1.

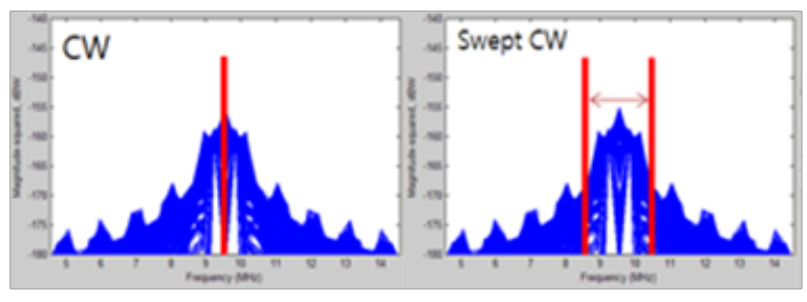

Fig. 1. Frequency response of the interference.

\section{INTERFERENCE DETECTION PARAMETERS}

\subsection{Adaptive Notch Filter Using RLS}

Analyzing and detecting the influence of interference is classified by two approaches; pre-correlation method and post-correlation method. The post-correlation method is a good approach to analyze the effect of the interference in terms of maintaining navigation solution quality. However, post-correlation methods cannot be used when a receiver does not track GNSS signal because the method uses the receiver measurements. In addition, post-correlation method requires more time to detect interference than pre-correlation method. Therefore, the pre-correlation method using adaptive notch filter is selected to detect the interference (Capozza et al. 2000, Chien et al. 2010) and is applied to multiple ground stations system in this paper.

An adaptive lattice IIR notch filter is used for the detection method, and the recursive least square (RLS) algorithm (Cho \& Lee 1993, Choi \& Cho 2002) is used to estimate frequency. The transfer function of the notch filter can be represented as

$$
H(z)=\frac{1-2 \cos \omega_{i} z^{-1}+z^{-2}}{1-2(1+r) \cos \omega_{i} z^{-1}+r z^{-2}}
$$

where $\omega_{i}$ determines the notch frequency, and is the distance between zero and pole. The frequency domain response of the transfer function is shown in Fig. 2. The transfer function of the notch filter consists of two parts, as shown in Fig. 3, where $a=\cos \left(\omega_{i}\right)$. The first part is called the auto-regression (AR) block, whose role is compensation of the moving average (MA) block (Borio et al. 2008). The

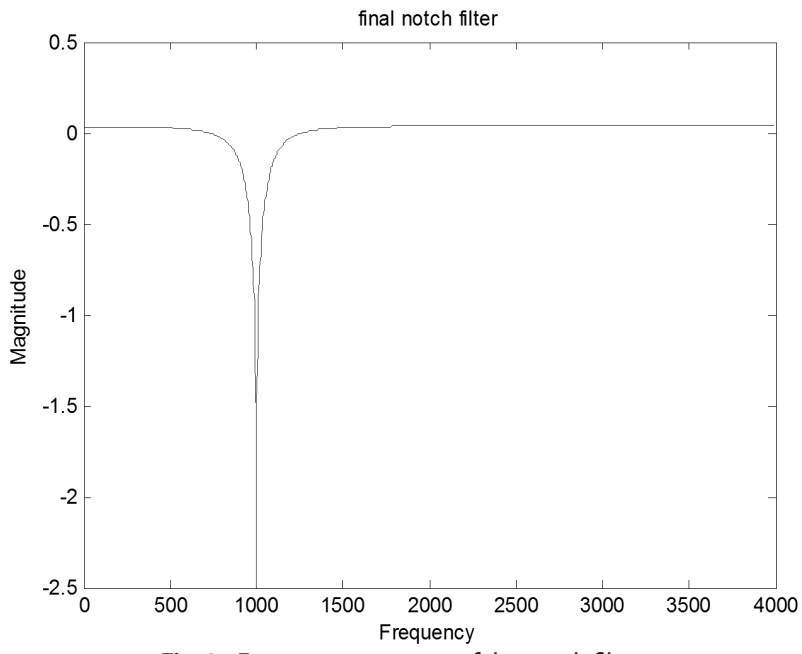

Fig. 2. Frequency response of the notch filter. 


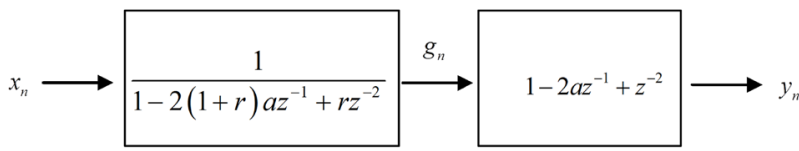

Fig. 3. Notch filter structure.
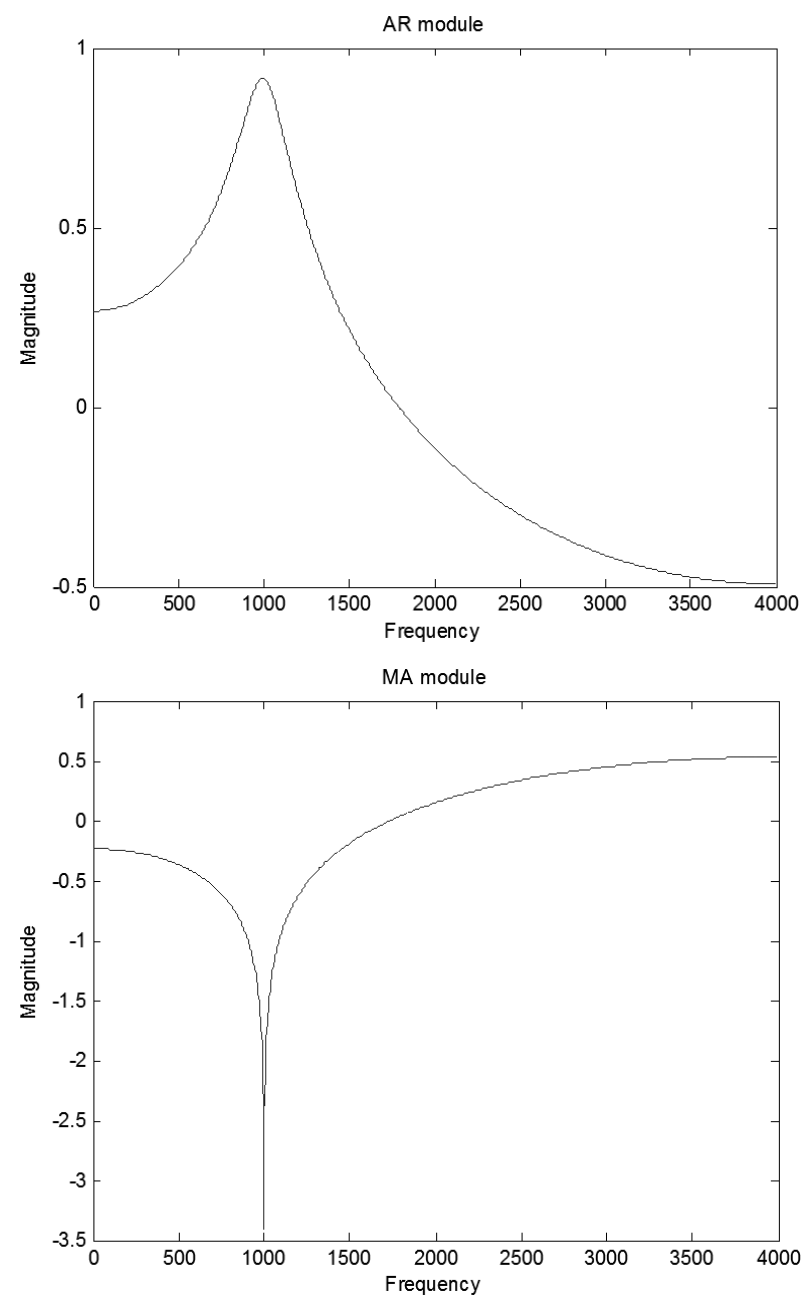

Fig. 4. Frequency response of the auto-regression block and the moving average block.

second block refers to the MA block, which can remove the signal located on the interference center frequency. The frequency domain responses of the two block output are shown in Fig. 4 respectively. When the notch is placed at the frequency of the interference, the output power is minimized. Therefore, the RLS algorithm uses the notch filter output power as a cost function,

$$
J=E\left[y_{n}^{2}\right]
$$

In order to find $a=\cos \left(\omega_{i}\right)$ which minimizes the cost function, the cost function is differentiated as

$$
\begin{aligned}
\frac{\partial J}{\partial a} & =E\left[2 y_{n}\left(-2 g_{n-1}\right)\right] \\
& =E\left[2\left(g_{n}-2 a g_{n-1}+g_{n-2}\right)\left(-2 g_{n-1}\right)\right]=0
\end{aligned}
$$

The adaptive algorithm for the estimation of frequency is summarized as follow: To obtain $a(n)$, Eq. (5) is rearranged as

$$
a(n)=\frac{E\left[g_{n-1}\left(g_{n}+g_{n-2}\right)\right]}{2 E\left[g_{n-1}^{2}\right]}=\frac{r_{1}(n)}{r_{0}(n)}
$$

$r_{0}(n), r_{1}(n)$ is defined as Eqs. (7) and (8) to perform RLS method.

$$
\begin{aligned}
& r_{1}(n)=\lambda r_{1}(n-1)+(1-\lambda)\left(g_{n-1}\left(g_{n}+g_{n-2}\right)\right) \\
& r_{0}(n)=\lambda r_{0}(n-1)+(1-\lambda) 2 g_{n-1}^{2}
\end{aligned}
$$

where $g_{n}$ is the output signal of the AR block and $\lambda$ is a forgetting factor, for recursive calculation.

For stability, the $a$ is clipped in the range of $[-1,1]$ and it is smoothed by

$$
\hat{a}(n)=\gamma \hat{a}(n-1)+(\gamma-1) a(n)
$$

where $\hat{a}(n)$ is the estimate of the $a(n)$, and $\gamma$ is the smoothing factor.

\subsection{Interference Detection Method Using Detection Parameters}

The proposed detection method shown in Fig. 5 consists of three parts which are adaptive notch filter explained previous section, detection logic and signal selection logic.

In general, the adaptive notch filter is able to reduce the effect of the CWI. However, the useful signal components near the interference center frequency are also removed because of the notch's bandwidth. To avoid this situation, the signal selection logic shown in Fig. 5 is added to the detection method, and only selects the filtered signal when

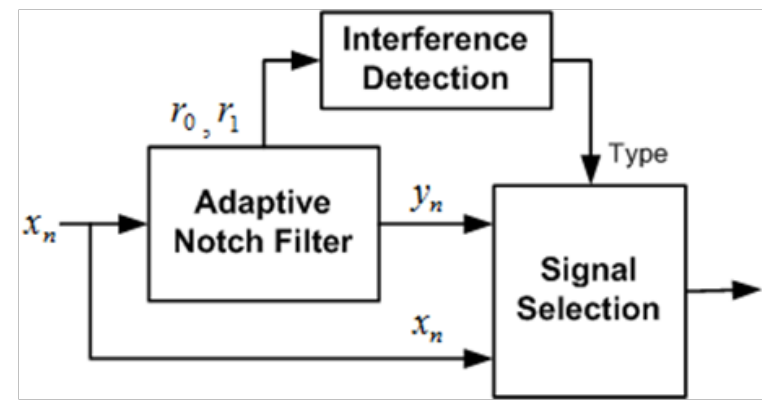

Fig. 5. Scheme of the interference detection method. 


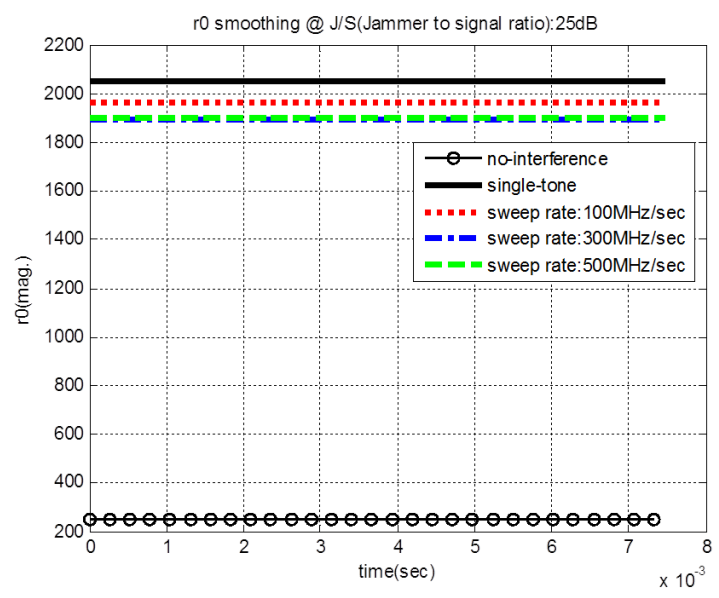

Fig. 6. Output of the detection parameter $r_{0}(n)$.

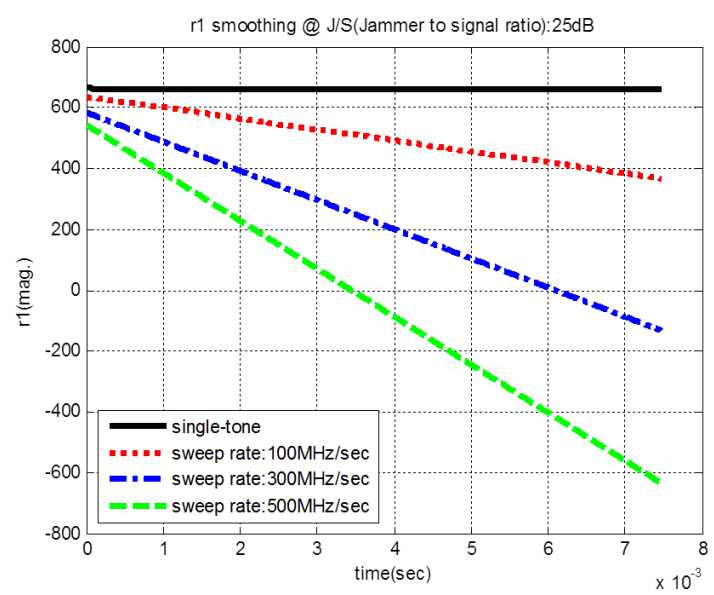

Fig. 7. Output of the detection parameter $r_{1}(n)$.

the interference signal is detected.

The interference detection logic uses the adaptive IIR notch filter parameters: $r_{0}(n), r_{1}(n)$, which are used to estimate the center frequency of the interference signal, and to derive the $\cos \left(\omega_{i}\right)$.

The parameters have unique characteristics, according to the type of incoming interference signal (Kang et al. 2012). When a single-tone CWI is received, $r_{0}(n)$ tends to converge to a certain value. If the interference signal power increases, the magnitude of $r_{0}(n)$ also increases, because it is the energy of the AR block output signal. Fig. 6 shows the characteristic of $r_{0}(n)$ with and without an interference signal. When the input signal is a swept CWI which linearly increases the center frequency of the interference, the value of $r_{1}(n)$ decreases linearly. This result is shown in Fig. 7. From the characteristics of $r_{0}(n)$ and $r_{1}(n)$, it is possible to detect interference signals, such as single-tone, and swept CWI.

The proposed detection logic is explained in Fig. 8. First, the mean value of $r_{0}(n)$ is calculated, and compared to a

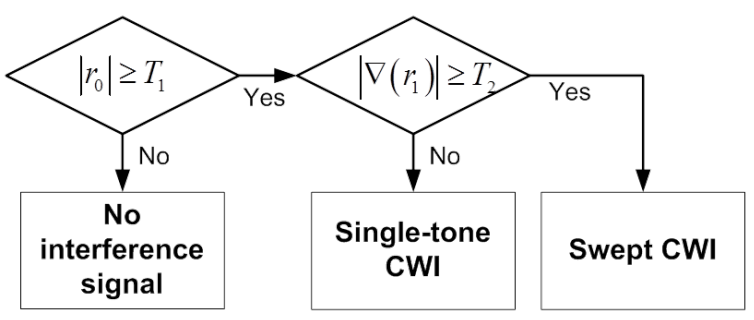

Fig. 8. Detection algorithm based on filter parameters.

threshold, to detect the interference signal. Second, the gradient of $r_{1}(n)$ is used to distinguish single-tone CWI and swept CWI. The process of the proposed method is as fallows. If $r_{0}(n)$ is below a threshold, the detection method classifies the received signal as no interference signal. If is above a threshold, the detection method compares the gradient of $r_{1}(n)$ to a threshold. If the gradient value is below the threshold, the detection method classifies the signal as singletone CWI. Otherwise, the signal is classified as swept CWI.

\section{SIMULATIONS}

The performance of the proposed detection method is verified by three scenarios. First, it is assumed that any interference signal is not received at first time, but few seconds later single-tone CWI is received. In the second scenario, the type of interference changes from single-tone CWI to swept CWI at few seconds later. In the last scenario, the proposed detection method is applied to the system constructed by multiple ground stations (Kang et al. 2012). In these scenarios, IF based GPS signal and interference are used at $9.548 \mathrm{MHz}$ and sampling frequency is $38.192 \mathrm{MHz}$. The power of interference is selected based on jammer to signal ratio (J/S) which refers to $25 \mathrm{~dB}$.

\subsection{Performance Analysis of interference Detection Method}

The results of interference identification in case of the first scenario are indicated in Fig. 9. The above figure refers to the characteristic of the parameter $r_{0}(n)$ explained in previous section. When the interference is received, the magnitude of converges to a certain value. The below figure indicates identification result of the received interference. If singletone CWI is received, the type of the interference indicates ' 1 '. In case of swept CWI, the type of the interference is ' 2 '. If there is no CWI, the type of CWI refers to '0'. The below figure in Fig. 9 presents the correct identification result of single-tone CWI which indicates ' 1 ' when the single-tone 

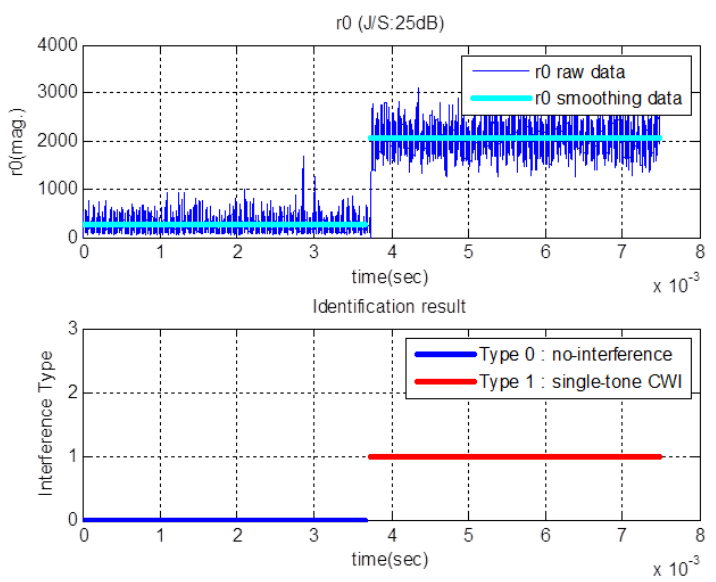

Fig. 9. Scenario 1: single-tone $\mathrm{CWI}$ detection result.
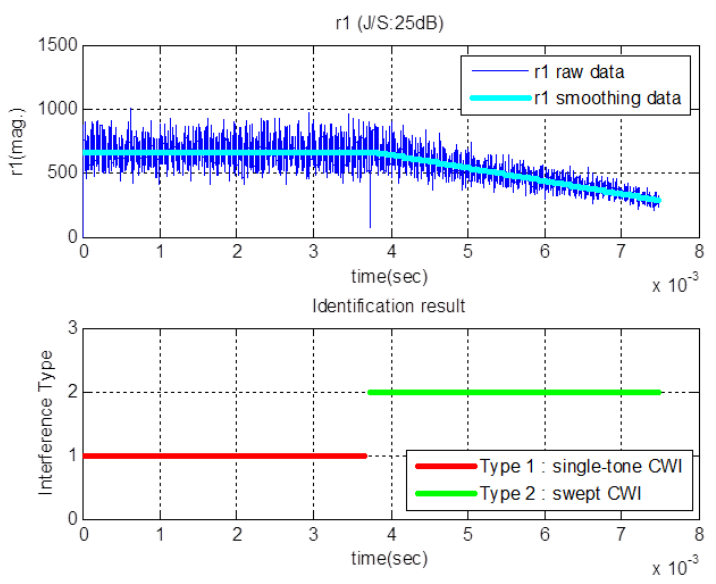

Fig. 10. Scenario 2: swept CWI detection result.

CWI is received. The proposed method is working well in the first scenario as shown in Fig. 9.

Likewise, Fig. 10 refers to the identification results of swept CWI. The above figure in Fig. 10 shows the characteristic of the parameter $r_{1}(n)$ whose gradient is related to the sweep rate as explained in the previous section. As shown in the below figure, the proposed interference detection method identifies the received interference as a swept CWI by indicating the type of the interference ' 2 ' only if the swept CWI is received. The Figs. 9 and 10 refer to the performance of detection method and the proposed method is working well in the scenarios.

The selection of the detection threshold has a great effect on the performance of the interference detection. In this paper, the threshold is selected to detect an interference signal whose $\mathrm{J} / \mathrm{S}$ is more than $25 \mathrm{~dB}$, which affects the accuracy of the GPS seriously. The detection probability has been calculated by Monte Carlo simulations, on the basis of 300 independent runs of the detection method. The detection rate of single-tone and swept CWI is perfect when the $\mathrm{J} / \mathrm{S}$ is higher than $25 \mathrm{~dB}$ which affects the navigation accuracy of the GPS.

\subsection{Performance Analysis of Interference Detection Method Applied to Multiple Ground Stations}

In this section, interference detection method is applied to multiple ground stations. The interference detection results (the type of interference: single-tone or swept) and power measurement of the interference from each ground station are used to obtain more precise information of the interference types. In this paper, the interference power measurements from each ground station are used to build weighting factors to estimate the types of interference more precisely. If one station's power measurement of the interference is higher than that of others, the weighting factor will be increased for identification of interference, because the accuracy of interference detection depends on the received power of interference (Kang et al. 2012). The detection result of the base station which has the biggest weighting factor is selected to make a final decision of the interference type.

The performance of interference detection algorithm is simulated for the scenarios of GPS signal in the presence of single-tone CWI. The interference transmit power is assumed to be $300 \mathrm{~mW}$ which is the power of commercial portable jammer. In addition, interference source characterization method is simulated with a set of three ground stations placed in a proposed arrangement shown in Fig. 11. Three base stations are arranged in the shape of a triangle in order to detect the interference which is in $7 \mathrm{~km}$ radius based on the main station, MS.

The proposed detection method is used for detecting a single-tone CWI and its location is fixed as represented in

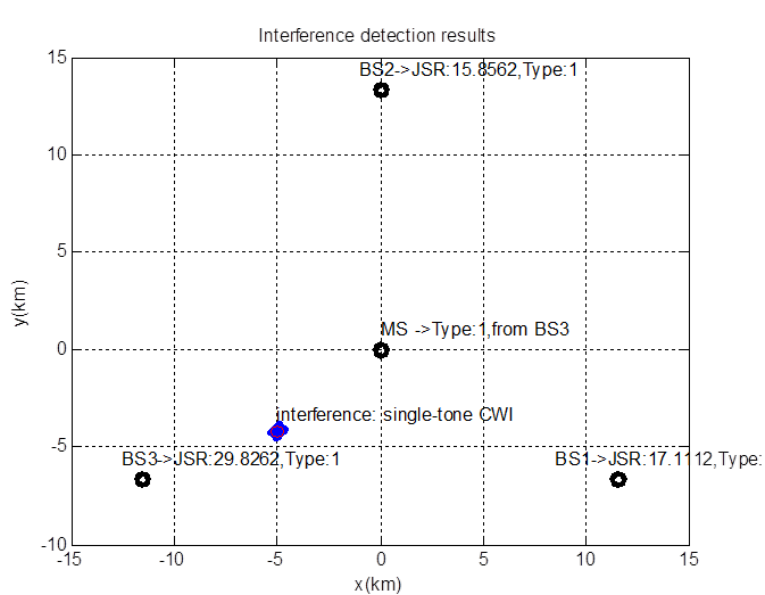

Fig. 11. Scenario 3: locations of the base stations and main station. 

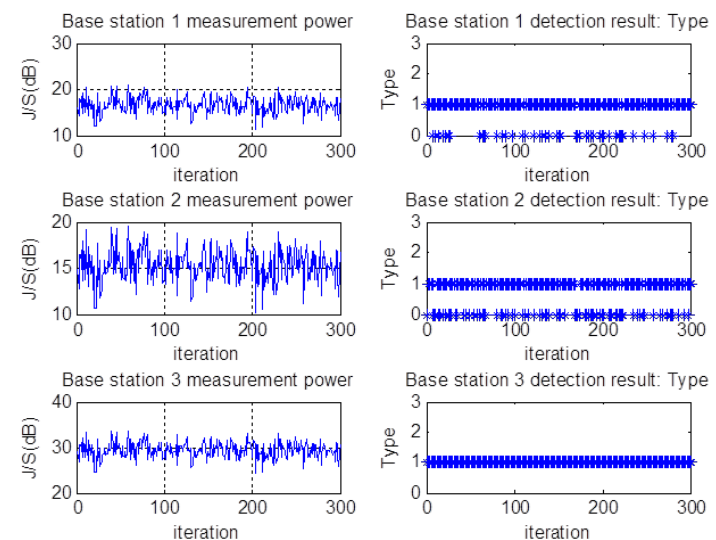

Fig. 12. Scenario 3: single-tone CWI detection results based on multiple ground stations.

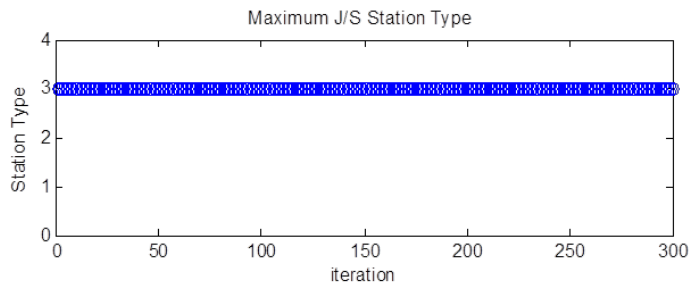

Main station detection result: Type

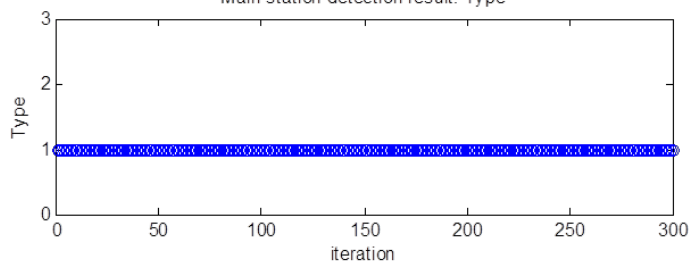

Fig. 13. Simulation 3: detection results of the main station.

Fig. 11. In addition, a generalized form for signal attenuation called Cost-231 Hata model (COST 1999) is used for simulation.

As shown in Fig. 11, the estimated J/S and interference identification result of each base station are expressed above the black mark of the base station. Blue background circle represents the location of the interference. The input type of interference is written above the blue circle. In addition, the final decision of interference identification is indicated as 'MS' and the ID of base station where the identification result of the interference are taken above the results of the base station (BS).

In order to verify the performance of the algorithm, the scenario is performed by Monte Carlo simulations on the basis of 300 iterations. In Fig. 12, the estimated J/S and Interference identification result of each base station are represented by iterations. Left side figures show that the estimated J/S and right side of figures refer to identification of the interference, which come up with in the same scenario shown in Fig. 11 which reflects $275^{\text {th }}$ iteration result of the Fig. 12. As shown in Fig. 12, the detection accuracy of BS 1 and BS 2 is relatively lower than that of BS3, because their estimated power is below $25 \mathrm{~dB}$ which is the reference power level for ensuring the perfect detection rate. The final decision of the main station in this scenario is shown in Fig. 13. In Fig. 13, the upper figure refers to the ID of base station where the identification result is taken, and the lower figure shows that the final identification results of the interference.

\section{CONCLUSION}

In this paper, detection method is proposed by using the characteristics of the adaptive filter output $r_{0}(n), r_{1}(n)$. When a single-tone CWI is received, $r_{0}(n)$ tends to converge to a certain value. In case of a swept CWI whose frequency is a linearly increasing, the value of $r_{1}(n)$ linearly decreases. Through the characteristics of parameters, it can detect a single-tone and swept CWI. The detection rate of single-tone and swept CWI is perfect when the J/S is higher than $25 \mathrm{~dB}$ which affects the navigation accuracy of the GPS. This paper also presents the proposed detection method is applied to multiple ground stations. The performance of the proposed method is evaluated by simulations of three scenarios. The simulation results show that the proposed method can efficiently detect single-tone and swept CWI. This proposed method is expected to be useful for identifying jamming situations, and for selecting effective interference mitigation methods.

\section{ACKNOWLEDGMENTS}

This work has been supported by the National GNSS Research Center program, of the Defense Acquisition Program Administration (DAPA) and Agency for Defense Development (ADD).

\section{REFERENCES}

Balaei, A. T., Dempster, A. G. 2009, A statistical inference technique for GPS interference dection, IEEE Trans. Aerosp. Electron. Syst., 45, 1499-1509

Betz, J. W. 2000, Effect of Narrowband Interference on GPS Code Tracking Accuracy, Proceedings of the Institute of Navigation 2000 National Technical Meeting, Inst. of Navigation, Anaheim, CA, 2000, pp.16-27

Borio, D., Camoriano, L., Presti, L. L. 2008, Two-Pole and Multi-Pole Notch Filters: A Computationally 
Effective Solution for GNSS Interference Detection and Mitigation, IEEE Systems Journal, 2, 38-47

Capozza, P. T. Holland, B. J., Hopkinson, T. M., Landrau, R. L., 2000, A single-chip narrow-band frequency-domain excisor for a Global Positioning System (GPS) receiver, IEEE J. Solid-State Circuits, 35, 401-411

Chien, Y. R., Huang, Y. C., Yang, D. N., Tsao, H. W. 2010, A Novel Continuous Wave Interference Detectable Adaptive Notch Filter for GPS Receivers, Global Telecommunications Conference (GLOBECOM 2010), Miami, FL, USA, Dec., 2010

Cho, N. I., Lee, S. U. 1993, On the adaptive lattice notch filter for the detection of sinusoids, IEEE Trans. Circuits Syst., 40, 405-416

Choi, J. W., Cho, N. I. 2002, Suppression of narrow-band interference in DS-Spread spectrum systems using adaptive IIR Notch filter, Signal Process., 82, 2003-2013

COST Action 231, 1999, Digital mobile radio towards future generation systems, final report, tech. rep., European Communities, EUR 18957

Kang, C. H., Yang, J. H., Kim, S. Y., Park, C. G. 2012, Detection and Characterization Algorithm of Swept Continuous Wave Interference for safe GBAS operation, Proc. ION GNSS 2012, Sep., 17-21 